CASE REPORT

\title{
"Fleck Sign": Traumatic Avulsion Fracture of the Medial Cunei- form by Anterior Tibialis Tendon
}

\author{
David H Kim ${ }^{1 *}$, Mark J Berkowitz ${ }^{2}$ and Jeff M Hrutkay ${ }^{3}$ \\ ${ }^{1}$ Colorado Permanente Medical Group, Lone Tree, Colorado, USA \\ ${ }^{2}$ The Cleveland Clinic, Cleveland, Ohio, USA \\ ${ }^{3}$ Colorado Permanente Medical Group, Lafayette, Colorado, USA
}

*Corresponding author: David H Kim, Colorado Permanente Medical Group, Lone Tree, Colorado, USA, E-mail: mbz81@yahoo.com

\section{Introduction}

We describe a case of avulsion of the tibialis anterior tendon in association with a complex midfoot fracture. Although cases of attritional tibialis anterior tendon ruptures exist in the literature, avulsion/rupture of the anterior tibialis in the setting of an isolated midfoot injury has not been previously reported in the English literature [1].

\section{Case Report}

The patient is a 45-year-old otherwise healthy male who was a helmeted motorcycle rider who struck a vehicle travelling at 30 miles per hour. The initial treating hospital had done a complete trauma work up, and concluded that his left foot injury was isolated. His past medical history was benign, and there had been no evidence of significant prior injury to the extremity. Physical examination in our office, approximately 1 week after injury, revealed superficial contusion (Oestern and Tscherne Classification 1), and swelling to the extremity without appreciable bump or irregularity at the dorsum of the foot. The isolated heel laceration had been stapled at the time of injury. Dorsiflexion was weak, but was felt to be due to pain and lack of effort. Intact sensation and less than 2 seconds of capillary refill were noted. Radiographs revealed fractures to the tarsal navicular and medial cuneiform, with subluxation of the first tarsometatarsal joint, as well as intercuneiform joint (Figure 1, Figure 2 and Figure 3). A small bony fragment "fleck" was seen on the oblique view of the foot (arrow, Figure 2). We did not feel that advanced medical imag-

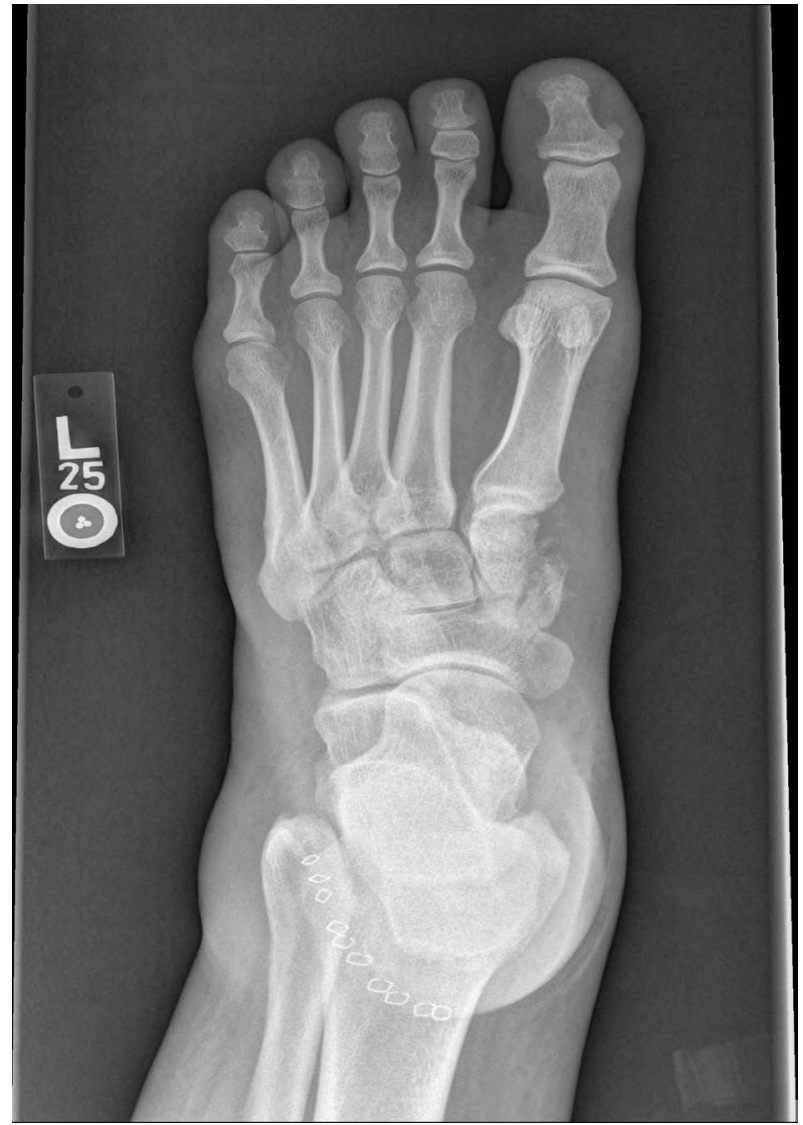

Figure 1: Injury AP foot radiograph, demonstrating medial cuneiform fracture. Staples which closed the soft tissue laceration are also seen.

ing would change our management of this patient, and would unnecessarily add to the cost of the treatment. 


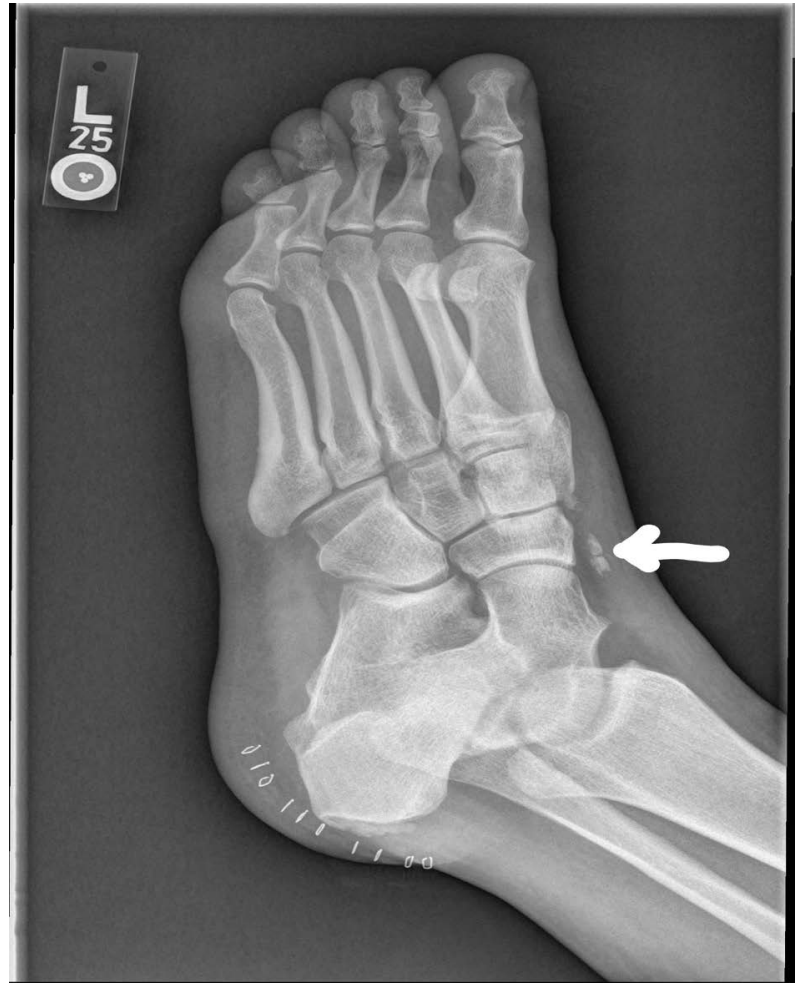

Figure 2: Oblique foot radiograph, clearly demonstrating the "fleck" sign, indicating the avulsion by the tibialis anterior tendon.

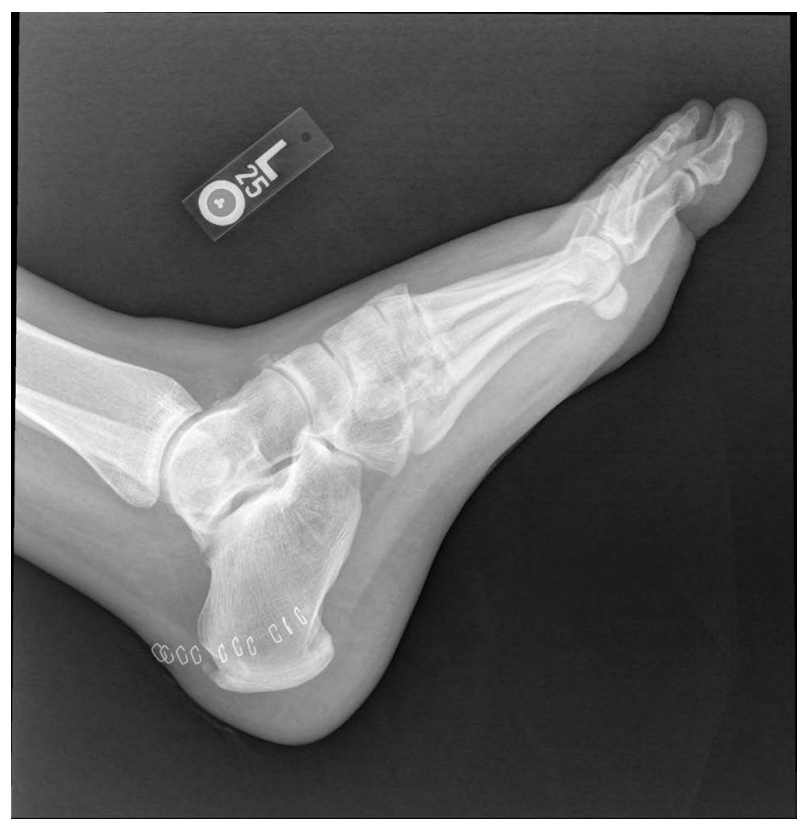

Figure 3: Lateral foot radiograph demonstrating the midfoot subluxation. "fleck" sign is not well seen.

Given the unstable nature of the injury, we counselled him for surgical intervention for fracture stabilization. Intra operatively, the absence of the tibialis anterior insertion was noted at the medial cuneiform, thus concomitant traumatic rupture of anterior tibialis was suspected. A separate incision was then made at the dorsal aspect of the foot, (Figure 4) where the small bony fragment "fleck" was confirmed to be the avulsion fracture fragment of the medial cuneiform, caused by the intact tibialis anterior ten-

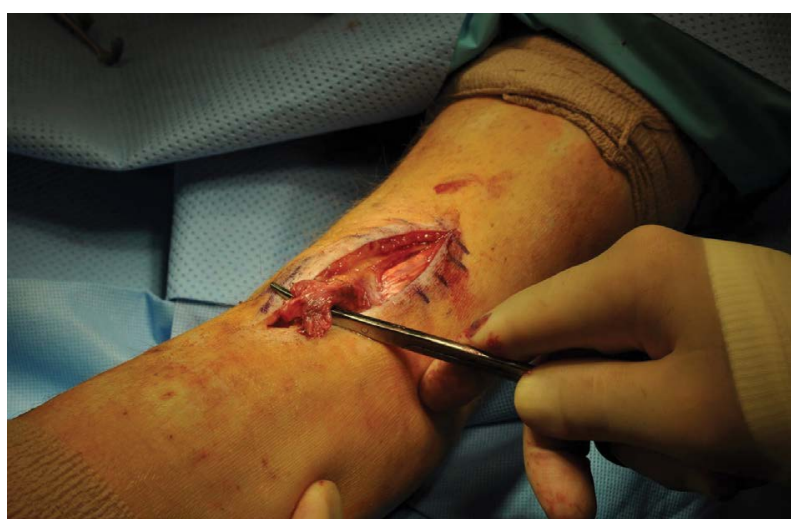

Figure 4: Intraoperative photograph demonstrating the injury.

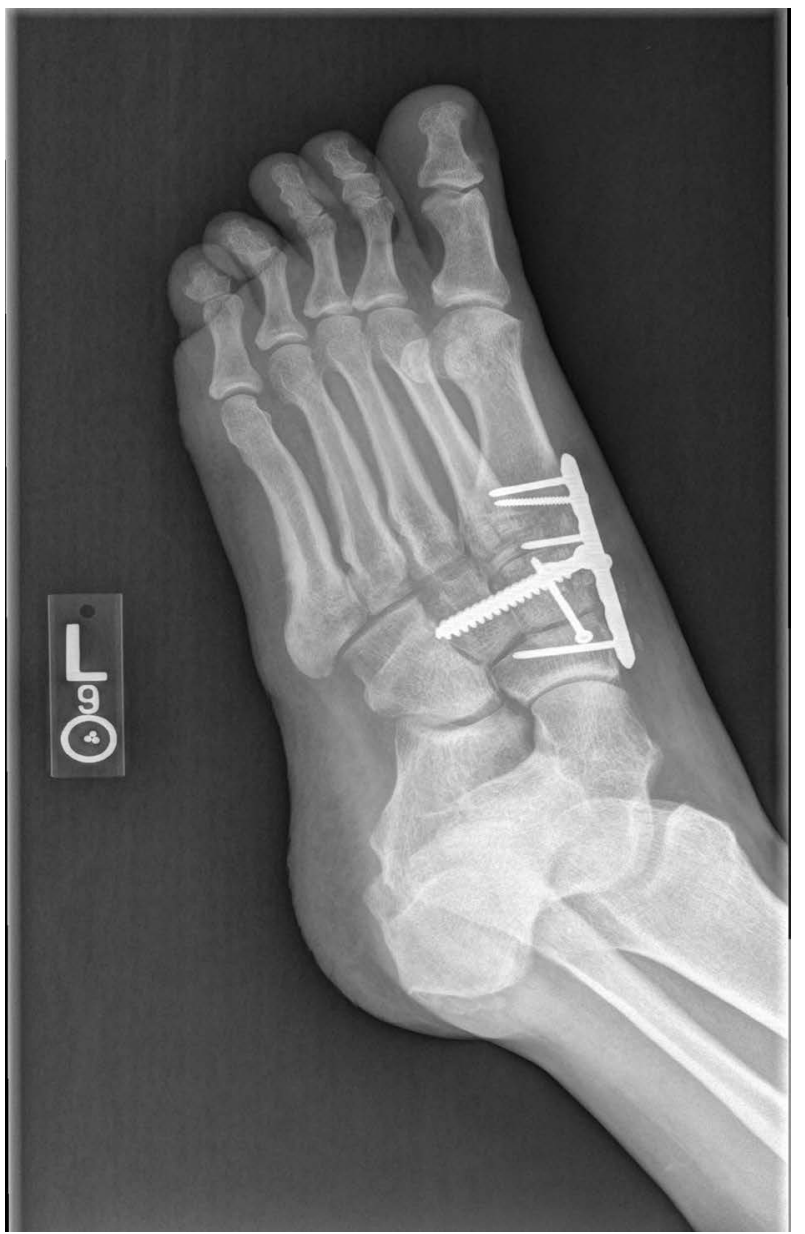

Figure 5: Post operative oblique foot radiograph.

don avulsing the bone. The small fragment of bone was excised, and the intact tendon was passedunder the extensor retinaculum. Reattachment to the medial cuneiform was accomplished by utilizing a spiked soft tissue washer over a $6.5 \mathrm{~mm}$ cancellous screw (Figure 5). Post-operatively, he was kept non-weight bearing for 8 weeks, followed by protected weight bearing. The patient recovered uneventfully, with some expected stiffness and pain at the final follow up at 12 months.

\section{Discussion}

The treatment of traumatic and atraumatic anteri- 
or tibialis tendon injuries has been well described in the literature $[2,3]$. These injuries typically occur in elderly patients with multiple co-morbidities, and not commonly seen in young patients involved in high energy injuries. Although tibialis anterior tendon interposition in association with Lisfranc injury can be encountered [4], avulsion of the medial cuneiform of the tibialis anterior is distinctly uncommon. Only one such case report in a non-English journal exists [1].

The original "fleck" sign was described by Myerson, et al. in the setting of tarsometatarsal joint fracture dislocations. This was felt to be present in $90 \%$ of the patients in their series in between the base of medial cuneiform and base of the second metatarsal [5]. Although the mechanism and the injury are obviously different, we felt that the radiographic appearance was similar to those which occur in tarsometatarsal injuries.

Mechrefe, Walsh, and DiGiovanni described avulsed anterior tibialis tendon which became entrapped in the fracture site of distal tibia preventing its reduction. After the tibia fracture was stabilized, the repair of the avulsed tendon was accomplished via using suture anchors [5]. No mention of pre-operative foot radiographs is included in their manuscript.

The patient described in our report likely sustained fractures of the medial cuneiform via direct blow at the time of the accident. In addition, due to the contraction of the anterior tibialis, a small piece of bone was avulsed from its insertion displacing it approximately $5 \mathrm{~cm}$ from its insertion [6].

We advocate a verycareful physical examination in the acute setting even if swelling and pain may make the examination suboptimal. The differential diagnoses for the "fleck" fragment seen on the oblique foot radiograph would include accessory ossicle and soft tissue calcifications. However, in the setting appropriate history, physical examination, along with plausible mechanism of injury, strong suspicion for tibialis anterior avulsion is warranted.

\section{References}

1. Ahn SH, Kim HC, Kim KY (2010) Avulsion fracture of medial cuneiform by tibialis anterior tendon. J Korean Foot Ankle Soc 14: 194-196.

2. Ouzounian TJ, Anderson R (1995) Anterior tibial tendon rupture. Foot Ankle Int 16: 406-410.

3. Sammarco VJ, Sammarco J, Henning C, Chaim S (2009) Surgical repair of acute and chronic tibialis anterior tendon ruptures. J Bone Joint Surg 91: 325-332.

4. Lowe J, Yosiphvitch Z (1976) Tarsometatarsal dislocation: a mechanism blocking manipulative reduction. Case report. J Bone Joint Surg 58: 1029-1030.

5. Mechrefe AP, Walsh EF, DiGiovanni CW (2006) Anterior tibial tendon avulsion with distal tibial fracture entrapment: Case report. Foot Ankle Int 27: 645-647.

6. Myerson MS, Fisher RT, Burgess AR, Kenzora JE (1986) Fracture dislocations of the tarsometatarsal joints: End results correlated with pathology and treatment. Foot Ankle 6: $225-242$ 